\title{
Condicionantes de consolidação de redes de cooperação interorganizacional: um estudo de caso sobre 0 Rio Grande do Sul
}

\author{
Claudio Zancan \\ Universidade Federal de Alagoas \\ Paulo da Cruz Freire dos Santos \\ Universidade Federal de Alagoas \\ Antônio Carlos Silva Costa \\ Universidade Federal de Alagoas \\ Nicholas Joseph Tavares da Cruz \\ Universidade Federal de Alagoas
}

\begin{abstract}
Este texto analisa a dinâmica de condicionantes envolvidos no processo de consolidação da rede Associação dos Produtores de Vinhos Finos do Vale dos Vinhedos (Aprovale) no setor vitivinícola brasileiro. A teoria consultada compreendeu abordagens organizacionais que definem o conceito de redes de cooperação como estratégia de coordenação de recursos interorganizacionais orientados em torno de objetivos comuns. Foram utilizados dois estudos: pesquisa documental e entrevistas. As conclusões indicaram que obtenção de economias de escala e reforço da capacidade competitiva constituíram os condicionantes principais neste processo. Estudos futuros são recomendados, analisando outros momentos evolutivos da rede, bem como condicionantes presentes em diferentes contextos e níveis de análise.
\end{abstract}

Palavras-chave: redes interorganizacionais; cooperação; setor vitivinícola brasileiro.

Factores determinantes de consolidación de redes de cooperación interorganizacionales: un estudio de caso sobre el Rio Grande do Sul

Este texto analiza la dinámica de condicionantes involucrados en el proceso de consolidación de la red Associação dos Produtores de Vinhos Finos do Vale dos Vinhedos (Aprovale), en Brasil. La teoría consultada comprendió abordajes organizacionales que definen el concepto de redes de cooperación como estrategia de coordinación de recursos interorganizacionales orientados en torno a objetivos comunes. Fueron utilizados dos estudios: investigación documental y entrevistas. Las conclusiones

Artigo recebido em 28 jul. 2012 e aceito em 18 fev. 2013.

Rev. Adm. Pública - Rio de Janeiro 47(3):647-669, maio/jun. 2013 
indicaron que la obtención de economías de escala y el refuerzo de la capacidad competitiva constituyeron los condicionantes principales en este proceso. Se recomienda estudios futuros que analicen otros momentos evolutivos de la red, bien como los condicionantes presentes en diferentes contextos y niveles de análisis.

Palabras clave: redes interorganizacionales; cooperación; vitivinicultura brasileña.

Determinants of consolidation of interorganizational cooperation network: a case study of the Rio Grande do Sul

This paper analyzes the dynamics of conditions involved in the consolidation process of the Association of Producers of Fine Wines from the Vineyards Valley at Brazilian wine industry. The theoretical basis used organizational approaches that define the concept of cooperation network as a coordination strategy of interorganizational resources oriented around common goals. The research comprises two qualitative studies: documentary analysis and interviews. It was used two studies: documentary research and interviews. The conclusions indicated that achieving economies of scale and enhanced competitiveness were the main conditions in this process. Further studies are recommended by analyzing evolution of the network in other times, as well as conditions present in different contexts and analysis levels.

KEYWORDs: interorganizational networks; cooperation; Brazilian wine industry.

\section{Introdução}

Aspectos relacionais evolutivos que envolvem o conceito de redes de cooperação interorganizacional conferem notável importância aos estudos organizacionais. Principalmente quando a dinâmica de relacionamentos presente nesses tipos de redes representa para o cenário empresarial algumas possibilidades estratégicas de acesso a novos mercados consumidores ou, em alguns casos, à manutenção de mercados atuais. Assim, o estudo do processo evolutivo das redes de cooperação tornou-se um tema recorrente entre estudiosos organizacionais, resultando na proliferação de proposições teóricas que analisam as diferentes etapas no gerenciamento do ciclo de vida das redes.

Estudos internacionais recentes como os de Müller-Seitz (2011), Lee e Monge (2011), Sirmon e colaboradores (2010), Atouba e Shumate (2010), Turrini e colaboradores (2009), entre outros, e nacionais como os de Poletto, Duarte e Mata (2011), Kunzler e Bulgacov (2011), Balestrin, Verschoore e Reyes Jr. (2010), entre outros, demonstram a importância das redes de cooperação como estratégias relacionais capazes de propiciar a geração de resultados que transcendem a simples soma dos recursos organizacionais individuais. Além disso, o compartilhamento de recursos e riscos, a sinergia resultante da interação organizacional e a estrutura de relacionamentos produzida proporcionam uma configuração de elementos que poderá resultar em aumento de competitividade para organizações que estabelecem redes de cooperação como alternativa de desenvolvimento (Powell, 1998).

Nesta direção, a pesquisa aqui relatada está inserida no âmbito de estudos que objetivam compreender a dinâmica relacional de condicionantes estratégicos que são acionados 
após o momento de formação das redes, objetivando sua consolidação, bem como o desenvolvimento de novas atividades compartilhadas entre as organizações participantes do processo cooperativo. Apesar da importância destacada na literatura para a estrutura interorganizacional denominada redes de cooperação, ainda pairam dúvidas sobre os reais motivos que levam algumas dessas redes a sobreviverem e prosperarem, enquanto outras não. Neste sentido, o objetivo deste trabalho foi descrever os condicionantes envolvidos durante o processo de consolidação da rede Associação dos Produtores de Vinhos Finos do Vale dos Vinhedos (Aprovale) no setor vitivinícola brasileiro.

Vale destacar que o objeto de estudo compreendeu uma rede produtiva que surgiu no cenário brasileiro em 1995, como uma associação comercial formada por um pequeno grupo de produtores localizados na região de Bento Gonçalves, no estado do Rio Grande do Sul. O surgimento dessa associação aconteceu em decorrência de dificuldades enfrentadas na geração individual de valor no mercado consumidor nacional de vinhos finos. As mudanças promovidas nas estruturas organizacionais destes cooperados, caracterizados como pequenos empresários (a maioria com um processo de gestão familiar, com baixo conhecimento tecnológico, baixas capacidades produtiva e de investimentos), resultaram, no final do ano de 2010, em uma estrutura de cooperação de grande destaque no setor vitivinícola nacional. Esse resultado demonstrou que a rede formada consolidou sua estrutura, contemplando, neste sentido, um conjunto de condicionantes plausíveis de observação com rigor científico.

Assim, para atender o objetivo estabelecido para este texto, o presente artigo está estruturado com mais cinco seções, além desta primeira, que é a introdução. Na segunda seção, como marco teórico, destacam-se: a evolução do conceito de redes de cooperação. A terceira seção trata da estrutura de condicionantes de consolidação dessas redes. A quarta seção é destinada ao detalhamento do método utilizado na pesquisa. Na quinta apresentam-se as análises dos dados. Por fim, a sexta seção é destinada às considerações finais e a sugestões para futuras pesquisas relacionadas com este tema em discussão.

\section{Redes de cooperação}

A intensificação da cooperação industrial tem estimulado a investigação dos fatores que afetam a amplitude e a complexidade das interdependências entre organizações, particularmente por meio da utilização de um recorte analítico baseado no conceito de redes de cooperação. Martes e colaboradores (2006:21) afirmam que "o conceito de redes de cooperação emergiu no final dos anos de 1970, quando autores como Aldrich e Williamson passaram a utilizar a forma de relacionamento interorganizacional como foco de análise". A influência dessa perspectiva fez com que o tema redes, neste início, fosse percebido a partir de diferentes enfoques. $\mathrm{O}$ primeiro enfoque considerava as redes de cooperação como um novo arranjo voltado à melhoria do desempenho organizacional. O segundo enfoque esteve voltado para o processo de formação e estruturação dos arranjos cooperativos. Por fim, o terceiro enfoque compreendeu 
os relacionamentos interorganizacionais cooperativos a partir de uma perspectiva temporal de forma mais ampla (Martes et al., 2006).

A partir destes três enfoques o conceito de redes de cooperação se desenvolveu, sendo abordado em diferentes perspectivas na literatura internacional. Por exemplo, publicações no Strategic Management Journal, no final dos anos de 1990, passaram a ser estruturadas por grupos específicos de abordagens, a saber: em um primeiro grupo foram reunidos trabalhos cujo tema central contempla a análise do desempenho das organizações envolvidas em algum tipo de cooperação interorganizacional, além da investigação sobre as vantagens competitivas decorrentes das estratégias de cooperação. O segundo grupo de trabalhos foi composto por artigos que tratam de motivos e condições necessárias para que as organizações adotem ações cooperativas, reunindo em seu entorno o maior número de publicações. Em um terceiro grupo foram reunidos artigos que propuseram e testaram modelos teóricos, considerando influências externas e internas do contexto de inserção das redes.

Também no final dos anos de 1990, o Academy of Maganagement Jornal apresentou evidências empíricas que tratavam da importância da formação das redes e alianças cooperativas no contexto interorganizacional. Entre os artigos publicados nesse journal ganharam destaque os trabalhos de Park e Ungson (1997) e os de Steensma e colaboradores (2000), que ressaltaram o aprendizado obtido por meio dos relacionamentos interorganizacionais como uma das principais razões que levam as organizações a adotarem estratégias cooperativas. Especificamente, no ano de 2004, por meio de uma edição especial, o Academy of Management Journal evidenciou aspectos inerentes ao desenvolvimento das estruturas das redes de cooperação como frameworks de evolução organizacional. Um exemplo que caracterizou bem essa ênfase foi o trabalho de Brass e colaboradores (2004), que abordou, entre outras questões, antecedentes e consequentes das redes de cooperação em vários níveis de análise.

Em eventos científicos internacionais, Balestrin, Verschoore e Reyes Jr. (2010) apresentaram evidências sobre o estudo das redes de cooperação nos últimos anos, entre elas: a) 15a edição do Association Internationale de Management Stratégique, realizado em 2006, que adotou como tema central as relações interorganizacionais; b) congresso da Academy of International Business (AIB), que ocorreu em 2008, em Milão, e apresentou como tema geral o desenvolvimento de conhecimento nas redes de negócios internacionais; e c) 15a edição do Multi-Organizational Partnerships, Alliances and Networks (Mopan), realizado em 2008, em Boston. Esses eventos científicos demonstraram, para esses autores, o notável interesse no estudo das redes de cooperação no contexto internacional.

No Brasil, também foi verificado interesse sobre o estudo das redes de cooperação, embora esse processo seja mais recente quando comparado com a academia internacional. O interesse na aplicação da perspectiva de redes no contexto dos estudos organizacionais brasileiros teve um aumento substancial na quantidade e na qualidade de pesquisas produzidas a partir da metade dos anos 2000 (Balestrin, Verschoore e Reyes Jr., 2010). Entre outras evidências tem-se o volume 46 da Revista de Administração de Empresas (RAE) em 2006, bem como o volume 43 da Revista de Administração Pública (RAP) em 2009. Ambos os periódicos, nesses volumes, adotaram a temática de redes como eixo principal de suas publicações. Além 
disso, eventos produzidos sob a coordenação da Associação Nacional de Pós-Graduação e Pesquisa em Administração (Anpad) passaram a contar com essa área temática em seis de suas divisões, em 2011: Administração da Informação, Administração Pública, Estudos Organizacionais, Estratégia em Organizações, Gestão de Ciência, Tecnologia e Inovação e Gestão de Operações Logísticas, consolidando dessa forma o crescimento significativo de interesse do tema no campo da administração no Brasil.

De forma geral, os resultados de pesquisas produzidas no Brasil sobre o enfoque temático de redes de cooperação podem ser percebidos em dois grupos. No primeiro grupo de resultados as redes são investigadas como alternativas estratégicas para a sobrevivência organizacional, revelando resultados que configuram tipos inovadores de alianças entre organizações (ou grupo de organizações) no gerenciamento dos relacionamentos interorganizacionais desenvolvidos. No segundo grupo de resultados estão situados aqueles estudos que consideram como objetivo a aplicação das técnicas de análise de redes sociais (ARS), ou seja, preocupam-se com a demonstração de resultados que revelem padrões estruturais dos relacionamentos organizacionais estabelecidos em forma de rede.

Apesar deste intenso volume de estudos sobre redes de cooperação nos cenários internacional e nacional, chega-se à conclusão de que no contexto interorganizacional brasileiro as redes têm sido apontadas como alternativas estratégicas para a sobrevivência empresarial. Nesse sentido, as redes consistem em um formato que configura uma distinta estrutura organizacional que ganhou notoriedade por combinar eficácia, informalidade e flexibilidade, rompendo com modelos ortodoxos de organização. Originadas de relacionamentos interorganizacionais, essas arquiteturas organizacionais expressam o grau de maturidade das articulações dos atores que as compõem, além das instituições em seu entorno, caracterizando um relacionamento ao mesmo tempo dinâmico e complexo (Ring e Van de Ven, 1994).

Portanto, neste artigo, entende-se que redes de cooperação podem ser vislumbradas como estruturas horizontais resultantes de relacionamentos interorganizacionais com ênfase no enfoque coletivo, comportando-se de maneira dinâmica na reconfiguração permanente de suas fronteiras, possibilitando melhor adaptação de recursos diante de objetivos estratégicos compartilhados entre as organizações que as formam. Dessa maneira, as redes podem ser consideradas entidades complexas, definidas como um arranjo único, cuja evolução depende, por um lado, da sua capacidade de facilitar a comunicação entre seus componentes e, por outro, da coerência entre seus objetivos com os seus componentes (Thompson, 2003).

Este conceito sugere que as redes de cooperação são estruturas interorganizacionais, capazes de superar limites durante sua trajetória evolutiva por meio da coordenação de recursos compartilhados, que viabilizam o fomento das atividades inovadoras e promovem sua evolução. Isto indica que, para o desenvolvimento interorganizacional ocorrer de forma satisfatória, tem-se a necessidade da existência de um conjunto de condicionantes favoráveis ao processo de consolidação das redes, provenientes do seu ambiente externo de inserção, além daqueles encontrados no contexto interno. Com o conhecimento prévio desses condicionantes, torna-se possível entender e gerenciar sua dinâmica de configuração, criando uma ambiência capaz de fazer as estruturas das redes consolidarem e prosperarem. 


\section{Condicionantes de consolidação de redes de cooperação}

No campo organizacional, os estudos que consideram as redes de cooperação como objetos de análise propõem diferentes momentos da variável temporal em torno de suas trajetórias evolutivas, ou seja, tais estudos tratam desde o período anterior à formação da rede até o momento de sua possível dissolução. Isso significa a ocorrência de fases distintas às redes de cooperação, quer seja no âmbito individual das organizações, quer seja no desenvolvimento das redes constituídas.

Ring e Van de Ven (1994) propuseram uma tipologia capaz de analisar as trajetórias de desenvolvimento das redes de cooperação definindo o processo evolutivo das mesmas como um fenômeno cíclico e não sequencial. A estratégia das organizações, a estrutura da indústria, o estágio tecnológico e o ciclo de vida dos produtos, para esses autores, fazem com que as redes de cooperação continuamente se modifiquem em um ciclo repetitivo de negociação, comprometimento e execução. Na negociação, as partes determinam conjuntamente oportunidades, riscos, objetivos, investimentos e ações que podem ser realizadas de forma cooperada. O comprometimento refere-se ao acerto entre as partes sobre o que fazer, como e quais mecanismos deverão ser utilizados para coordenar as atividades. Por fim, a execução refere-se à colocação em prática dos acertos realizados nas etapas anteriores (Ring e Van de Ven, 1994).

Franco (2007) especificou três diferentes e inter-relacionadas fases que constituem uma rede de cooperação em torno de sua trajetória de evolução: formação, consolidação e desenvolvimento. Na fase de formação ocorre a descoberta, a exploração de oportunidades de colaboração, bem como as negociações entre as empresas cooperantes. Ainda nessa fase, a identificação inicial das oportunidades baseada na validação de ideias empresariais, objetivos claros, limites de recursos internos e externos dos atores e uma atenção significativa na eleição e tipo de parceiro são aspectos que devem ser considerados para reduzir potenciais dificuldades entre as partes envolvidas.

Na consolidação, as características e os objetivos da colaboração são determinados e os meios disponíveis para a consecução da rede são estabelecidos. No entanto, os parceiros podem ainda ter interesses divergentes, dependendo de fatores internos (dimensão, antiguidade, estratégia, caráter familiar, tecnologia, entre outros) e externos (cultura, setor de atividade, mercado, concorrência, entre outros) que envolvem a rede.

Por fim, Franco (2007) considerou a fase de desenvolvimento, tendo em conta que o sucesso, a estabilidade e os resultados da colaboração entre empresas são componentes importantes. Afirma esse autor: "se o sucesso e a estabilidade forem alcançados em uma rede de cooperação, os parceiros competem mais eficiente e eficazmente e, por conseguinte, o desempenho, a satisfação e o processo de aprendizagem serão melhores, proporcionando o desenvolvimento da rede" (Franco, 2007:157).

Especificamente, contemplando a coordenação de recursos no processo de consolidação das redes de cooperação — foco deste artigo — , percebe-se na literatura consultada que 
as abordagens teóricas que analisam a fase de consolidação das redes de cooperação têm demonstrado que a mesma ocorre com base em modelos descritivos (Criado e Criado, 1996; Murray, 1995, entre outros). Esses modelos analisam características e objetivos da cooperação das redes para verificar sua consolidação, abordando o conteúdo da cooperação (domínio, orientação e tipos de transações), especificidades contratuais (formas e período de duração) e sobre sua organização (grau de formalização, número das organizações envolvidas e aspectos geográficos).

Em outra perspectiva, Lee e Monge (2011) argumentam que a bibliografia nem sempre é suficientemente específica sobre o assunto que envolve a consolidação das redes de cooperação e, desse modo, as generalizações são comuns. Todavia, Ring e Van de Ven (1994) entendem que alianças estratégicas, redes de cooperação, joint ventures, consórcios de investigação e outras formas de redes organizacionais são dependentes de acordos contratuais complexos direcionados à consecução de objetivos estratégicos para obterem sua consolidação.

Dessa forma, as abordagens clássicas e recentes que fazem referências à consecução de objetivos estratégicos na consolidação da cooperação organizacional têm examinado este processo com base em diferentes perspectivas, entre elas: a) compartilhamento de riscos (Poletto, Duarte e Mata, 2011; Mowery, Oxley e Silverman, 1996; Sachwald, 1998); b) racionalização da produção e economias de escala (Posch, 2010; Glaister e Buckley, 1996; Kent, 1991); c) expansão internacional (Chen, 2010; Hausman et al., 2010; Merchant e Schendel, 2000); d) transferência de tecnologias (Saeed, Malhotra e Grover, 2011; Ricks, 1993); e e) complementaridade de recursos (Kunzler e Bulgacov, 2011; Lee e Monge, 2011).

Estas abordagens aludem a alguns dos objetivos a alcançar com a cooperação no contexto empresarial. Também outros objetivos podem ser considerados para justificar a consolidação das redes de cooperação. Por exemplo, para Franco (2007), as redes de cooperação podem criar vantagens competitivas quer no nível da diferenciação, quer no nível dos custos. Para Glaister e Buckley (1996), os relacionamentos cooperativos permitem diversificar áreas atrativas do negócio. A partilha dos custos fixos é outro objetivo que tem permitido a concretização de redes de cooperação entre organizações empresariais (Ohmae, 1990). Sob outra perspectiva, Poletto, Duarte e Mata (2011) ressaltam a importância da verificação de objetivos estratégicos relacionados à criação de conhecimento e inovações tecnológicas, principalmente quando esse conhecimento é associado às inovações tecnológicas necessárias na obtenção de vantagem sustentável na rede.

No entanto, apesar da existência de certo consenso entre os estudiosos a respeito da importância desses objetivos como elementos indispensáveis ao processo de consolidação das redes, sua dinâmica relacional não aparece de maneira detalhada na literatura. De fato, existem muitas razões que permitem a consolidação das redes de cooperação acontecer, mas os estudos produzidos disponíveis dão pouca indicação do que se espera alcançar, em termos de importância relativa do conjunto de objetivos. Isso decorre porque quando uma rede de cooperação é estabelecida, diversos objetivos podem estar presentes, não apenas um. 
Nesse sentido, a percepção e a discussão de como esses objetivos podem estar relacionados no processo de consolidação das redes de cooperação induzem a proposição de uma estrutura de condicionantes capazes de contribuir com sua consecução durante a trajetória evolutiva das redes, o que já foi observado nos estudos discutidos neste referencial, entre eles: Poletto, Duarte e Mata (2011), Glaister e Buckley (1996) e Saeed, Malhotra e Grover (2011).

Isso permite, desse modo, que os condicionantes possam ser passíveis de investigação de forma teórica no ambiente das redes. Portanto, a importância que os mesmos assumem no processo de consolidação para a definição dos objetivos estratégicos das redes influenciou o método de pesquisa utilizado no trabalho cujos resultados aqui serão relatados. O quadro 1 sintetiza os principais condicionantes abordados, bem como os autores relacionados como base teórica.

Quadro 1

\section{Condicionantes de consolidação de redes de cooperação}

\begin{tabular}{|c|c|}
\hline Fatores condicionantes & Autores relacionados \\
\hline Competências e aprendizagem & Poletto, Duarte e Mata (201 1) e Saeed, Malhotra e Grover (2011) \\
\hline Criação de vantagens competitivas & Sirmon e colaboradores (2010) e Glaister e Buckley (1996) \\
\hline Partilha de custos fixos & Bleeke e Ernst (1992) e Ohmae (1990) \\
\hline $\begin{array}{l}\text { Compartilhamento de riscos } \\
\text { operacionais }\end{array}$ & Mowery, Oxley e Silverman (1996) e Sachwald (1998) \\
\hline Racionalização da produção & Posch (2010) e Glaister e Buckley (1996) \\
\hline Economias de escala & Posch (2010), Glaister e Buckley (1996) e Perrow (1992) \\
\hline Expansão internacional & Chen (2010), Hausman e colaboradores (2010) e Merchant e Schender (2000) \\
\hline Transferências de tecnologias & Saeed, Malhotra e Grover (2011), Sachwald (1998) e Ricks (1993) \\
\hline Complementaridade de recursos & Kunzler e Bulgacov (2011), Lee e Monge (2011) e Müller-Seitz (2011) \\
\hline Confiança & Thorgren e Wincent (2010) e Perrow (1992) \\
\hline $\begin{array}{l}\text { Reforço de capacidades competitiva } \\
\text { e produtiva }\end{array}$ & $\begin{array}{c}\text { Sirmon e colaboradores (2010), Mowery, Oxley e Silverman (1996) e Sachwald } \\
\text { (1998) }\end{array}$ \\
\hline
\end{tabular}

Fonte: Elaborado pelos autores.

\section{Método}

Para atender o objetivo principal aqui proposto - descrever os condicionantes envolvidos durante o processo de consolidação da rede Aprovale no setor vitivinícola brasileiro — foram utilizados dois procedimentos: pesquisa documental e entrevistas, sendo classificado como 
um estudo descritivo-comparativo. O nível de análise foi o de redes e as unidades de análises, os relacionamentos cooperados estabelecidos entre organizações associadas à rede em estudo e entidades representativas do setor vitivinícola brasileiro durante o momento de consecução dos objetivos estabelecidos no processo de formação dessa rede.

A pesquisa documental adotou dois momentos. No primeiro momento foram investigadas informações sobre o tema "Redes de Cooperação", identificadas por meio de pesquisa efetuada nas bases de dados do Business ABI/Inform Global e do Business Source Complete, utilizando-se os termos: interorganizational network e cooperation; social network e cooperation network, cooperation network e collaborative; e innovation network e cooperation. Esses termos deveriam estar presentes em títulos, palavras-chave ou em abstracts de artigos completos, com dados empíricos, publicados em periódicos acadêmicos, no período de janeiro de 2000 a dezembro de 2011. Utilizando os mesmos critérios do levantamento anterior, com palavras-chave em português, realizou-se busca nos principais periódicos brasileiros de Administração (RAE, RAE-Eletrônica, RAC, RAP, Rausp, O\&S e Read), bem como em teses dos programas de pós-graduação reconhecidos pela Capes com conceito igual ou superior a cinco. Como resultado desta etapa, foram construídas as categorias teóricas utilizadas no estudo, conforme pode ser observado, resumidamente, no quadro 1 deste texto.

No segundo momento da pesquisa documental foram examinados os sítios eletrônicos do Instituto Brasileiro do Vinho (IBV), da Aprovale e da Embrapa Uva e Vinho com o objetivo de encontrar informações relevantes que envolviam o contexto analisado. Para a análise dos dados obtidos no segundo momento da pesquisa documental, após uma revisão detalhada dos documentos encontrados, os mesmos foram agrupados e resumidos em três categorias: a vitivinicultura no cenário nacional, no estado do Rio Grande do Sul e na região do Vale dos Vinhedos. É válido ressaltar que, por motivos de espaço, neste artigo, são apresentados apenas os resultados encontrados no terceiro tópico que envolveu a região do Vale dos Vinhedos, com especificidade na Aprovale. A importância da apresentação destas informações está na contextualização do cenário que envolveu a formação da rede analisada.

Na realização das entrevistas, foram ouvidos nove gestores de vinícolas associadas à rede estudada, além de três representantes de organizações envolvidas diretamente no processo de consolidação dessa rede, entre os meses de janeiro e abril de 2010. O conteúdo das questões utilizadas nas entrevistas versou sobre o processo evolutivo das redes de cooperação, com ênfase no modelo proposto por Franco (2007), que contempla mecanismos de coordenação de recursos das redes de cooperação. O quadro 2 relaciona a organização entrevistada, os cargos dos entrevistados e o tempo de duração das entrevistas. 
Quadro 2

\section{Dados das entrevistas}

\begin{tabular}{|lcc|}
\hline Organização entrevistada & Cargo do respondente & Tempo de duração \\
\hline Casa Valduga & Gerente de marketing & 75 minutos \\
Chandon do Brasil & Diretor-geral & 91 minutos \\
Cooperativa e Vinícola Aurora & Gerente de marketing & 63 minutos \\
Vinícola Miolo & Gerente de marketing & 84 minutos \\
Vallontano Vinhos Nobres & Proprietária - diretora comercial & 100 minutos \\
Vinícola Boutique Lídio Carraro & Proprietária - diretora comercial & 61 minutos \\
Vinícola Cordelier & Diretor industrial & 64 minutos \\
Vinícola Dom Cândido & Proprietário - diretor administrativo & 68 minutos \\
Vinícola Marco Luigi & Proprietária - diretora administrativa & 86 minutos \\
Ibravin & Gerente de marketing & 125 minutos \\
Gerência Executiva Aprovale & Diretor executivo & 112 minutos \\
Embrapa Uva e Vinho & Pesquisador responsável na Aprovale & 96 minutos \\
\hline
\end{tabular}

Fonte: Elaborado pelos autores.

Para o tratamento dos dados obtidos nas entrevistas foi utilizada a técnica de análise de conteúdo do tipo temática com codificação axial. O tratamento de dados por meio do processo de codificação axial consiste na redução de informações com base em códigos previamente definidos constantes na literatura. Os códigos utilizados nesse processo foram os mesmos apresentados no quadro 1. Com a utilização do software Atlas TI, foram seguidos três passos para o tratamento dos dados. Primeiramente, os documentos primários foram criados e preparados com a transcrição do texto das entrevistas. No segundo passo, as unidades de significação adotadas (códigos) foram relacionadas com as partes mais importantes do conteúdo das entrevistas que estavam relacionadas com as mesmas. Isso gerou uma tabela de frequência com o número de vezes que o código era abordado a partir do documento primário, adicionado do trecho do documento primário (parte da entrevista) que comprovava a relação. Como um terceiro passo, os códigos foram relacionados de acordo com a dinâmica de aparecimento dos mesmos, gerando graficamente uma teia de relações entre os códigos adotados, além da descrição dos acontecimentos que levaram à possível consolidação da rede em estudo.

Assim, têm-se as principais características metodológicas utilizadas para a consecução do objetivo principal deste texto. Na próxima seção serão apresentados os resultados. Vale ressaltar que este texto é oriundo de um estudo maior que analisou a trajetória evolutiva da 
Aprovale entre 1995 e 2010. Neste artigo são apresentados apenas alguns dos resultados relevantes encontrados.

\section{Apresentação e discussão de resultados}

Neste tópico são apresentados e discutidos os resultados encontrados por meio da aplicação do método utilizado na pesquisa. Especificamente, são demonstrados como resultados algumas características gerais do Vale dos Vinhedos e da rede Aprovale (conteúdo do segundo momento da pesquisa documental), bem como os resultados que envolveram os condicionantes de consolidação responsáveis pelo processo de estabilização e fortalecimento da rede analisada (conteúdo das entrevistas).

\section{1. Características gerais do Vale dos Vinhedos e da Aprovale}

O Vale dos Vinhedos é um território localizado na Serra Gaúcha, mais precisamente na região nordeste do estado do Rio Grande do Sul. A composição geográfica se dá pela intersecção dos municípios de Bento Gonçalves, Garibaldi e Monte Belo do Sul. Trata-se de uma área caracterizada por uma bacia hidrográfica com vários riachos e pequenos rios que banham uma área montanhosa de $81.123 \mathrm{~km}^{2}$. A gênese de seu povoamento se deu basicamente por meio da colonização italiana por volta de 1875 . Os imigrantes italianos eram, em sua maioria, oriundos da região do Vêneto e Trento (Aprovale, 2011).

Com base em dados obtidos na Embrapa Uva e Vinho (Embrapa, 2011), atualmente, do total da área do Vale dos Vinhedos, em $26 \%$ são encontrados vinhedos, em 43\%, florestas, e $31 \%$ está destinado para plantio de outras culturas. Ainda com base nesses dados, tem-se que, em relação ao uso do solo, 10\% podem ser caracterizados como de uso tipicamente urbano em área rural. O Vale dos Vinhedos está localizado a pouco mais de $120 \mathrm{~km}$ de Porto Alegre, capital estadual do Rio Grande do Sul. A região do Vale dos Vinhedos apresenta clima temperado, possui quatro estações bem definidas e sua paisagem muda em conformidade com as estações do ano. Quando ocorrem invernos rigorosos, para os padrões brasileiros, a indústria do turismo instalada nas proximidades do vale costuma explorar sua presença atraindo turistas e, indiretamente, beneficia a divulgação do Vale dos Vinhedos.

De acordo com informações extraídas do sítio eletrônico do Instituto Brasileiro do Vinho (Ibravin, 2011), nota-se que o território do Vale dos Vinhedos é caracterizado como um espaço da agricultura familiar. Isso é caracterizado pela existência de 375 famílias de viticultores cadastrados no Vale dos Vinhedos. Essas famílias costumam se organizar em forma de núcleos, ou seja, cada produtor se associa a uma vinícola, geralmente aquela que se encontra mais próxima a sua propriedade, e fornece sua produção de uvas para a vinícola parceira industrializar. 
É neste cenário que surge a rede Aprovale. No entanto, vale ressaltar que o processo de formação da rede não surgiu de forma espontânea entre os produtores localizados nesta região. Um dos fatores que propiciou o surgimento da mesma aconteceu nas décadas de 1960 e 1970, quando grandes empresas multinacionais de bebidas alcoólicas entraram no mercado brasileiro, elevando o grau de competitividade para as organizações que pertenciam ao setor vitivinícola. Nesse período, as vinícolas localizadas no Vale dos Vinhedos não estavam satisfeitas com os baixos desempenhos que envolviam seus negócios e o setor vitivinícola como um todo. Era evidente que alguma coisa precisava ser feita para que os produtos provenientes dessa região ganhassem competitividade e mercado. Portanto, uma associação cooperativa parecia ser uma alternativa estratégica plausível para reverter a condição precária que permeava os negócios desses produtores (Fensterseifer, 2007).

Assim, em 1995, sete vinícolas fundaram a Aprovale: Vinícola Miolo Ltda., Luiz Valduga \& Filhos Ltda., Casa Cordelier Ind. de Bebidas Ltda., Vinhos Don Laurindo Ltda., Adega Cavalleri Ltda., Adega de Vinhos Finos Dom Cândido Ltda. e Vinho Casa Graciema Ltda. Pelo que se observa no sítio eletrônico da associação Aprovale, o principal objetivo de sua criação consistiu em formar no Brasil a primeira Indicação de Procedência (IP) para vinhos finos e espumantes, bem como transformar a região do Vale dos Vinhedos em uma rota do Enoturismo nacional (Aprovale, 2011).

Após um processo que perdurou cerca de oito anos, a partir da criação da associação em 22 de novembro de 2002, o Vale dos Vinhedos tornou-se a primeira região do Brasil a obter uma Indicação de Procedência (IP) de vinhos finos e espumantes reconhecidas pela União Europeia (Aprovale, 2011). Entre os atores institucionais envolvidos durante o processo de reconhecimento geográfico que foram responsáveis pela elaboração e detalhamento de estudos técnicos estão: Empresa Brasileira de Pesquisa Agropecuária (Embrapa Uva e Vinho), Universidade Federal do Rio Grande do Sul (UFRGS) e Universidade de Caxias do Sul (UCS).

O titular da IP Vale dos Vinhedos é a Aprovale, que no final de 2010 contava com 31 vinícolas associadas e 39 associados não produtores (queijarias, hotéis, restaurantes, pousadas, entre outros). Dados da produção de 2010 mostram que as vinícolas do Vale dos Vinhedos produziram um volume de 10,1 milhões de garrafas de vinhos finos e espumantes, e esse volume de produção significa em torno de $20 \%$ da produção de vinhos finos e $35 \%$ da produção de espumantes, do total da produção desses produtos que são oriundos do estado do Rio Grande do Sul (Aprovale, 2011).

Dessa maneira, é com base na importância econômica e social que esta rede representa para o setor produtivo brasileiro que os condicionantes responsáveis por sua consolidação ganharam relevância, podendo esses resultados inspirar a definição de objetivos estratégicos que levem à consolidação de outras redes produtivas no Brasil. No próximo tópico serão apresentados os resultados da análise dos condicionantes. 


\subsection{Condicionantes de consolidação da Aprovale}

No processamento dos dados, os achados encontrados que abrangem a dinâmica dos condicionantes responsáveis pela consolidação da rede Aprovale foram especificados em dois grupos de resultados principais: o primeiro grupo demonstra, por meio de uma tabela de frequência, a incidência dos condicionantes relacionados à fase de consolidação da Aprovale; o segundo grupo, por sua vez, proporciona a geração de uma teia de relacionamentos entre os condicionantes evidenciados com base nas informações obtidas por meio das entrevistas entre os respondentes especificados. A tabela 1 sintetiza os dados relacionados com o primeiro grupo de resultados.

\section{Tabela 1}

Incidência de condicionantes na consolidação da Aprovale

\begin{tabular}{|lccc|}
\hline \multicolumn{1}{|c}{$\begin{array}{c}\text { Condicionantes identificados } \\
\text { na consolidação da Aprovale }\end{array}$} & $\begin{array}{c}\text { Códigos } \\
\text { (Codes) }\end{array}$ & $\begin{array}{c}\text { Total de incidência dos } \\
\text { condicionantes }\end{array}$ & $\begin{array}{c}\text { Percentual de incidência dos } \\
\text { condicionantes }\end{array}$ \\
\hline Economias de Escala & Code78 & 46 & $12,53 \%$ \\
Criação de Vantagem Competitiva & Code67 & 41 & $11,17 \%$ \\
Reforço da Capacidade Produtiva & Code75 & 39 & $10,62 \%$ \\
Expansão Internacional & Code70 & 37 & $10,08 \%$ \\
Compartilhamento de Custos e Riscos & Code68 & 36 & $9,80 \%$ \\
Reforço da Capacidade Competitiva & Code76 & 36 & $9,80 \%$ \\
Complementaridade de Recursos & Code72 & 31 & $8,44 \%$ \\
Transferência de Tecnologias & Code71 & 27 & $7,35 \%$ \\
Racionalização da Produção & Code69 & 23 & $6,26 \%$ \\
Aprendizagem & Code77 & 19 & $5,17 \%$ \\
Desenvolvimento de Competências & Code66 & 14 & $3,81 \%$ \\
Bem-estar Social & Code74 & 10 & $2,72 \%$ \\
Confiança & Code73 & 08 & $2,17 \%$ \\
\hline
\end{tabular}

Fonte: Dados da pesquisa.

Com base nestes resultados apresentados, percebe-se que os condicionantes mais citados foram: economias de escala (46 incidências) e criação de vantagem competitiva (41 incidências). Vale destacar que, embora os principais produtos da rede analisada, vinhos finos e 
espumantes, constituam produtos com alto valor agregado, contemplando valores simbólicos e únicos, nota-se de forma objetiva que os associados da Aprovale buscaram no processo de consolidação da rede, prioritariamente, obter ganhos de escala como estratégia para tornar seu negócio sustentável, ou seja, quantidade produzida com foco na diferenciação da produção como ferramenta de inserção e ganho de mercado.

Já os condicionantes que obtiveram um menor número de incidências foram: confiança (oito incidências) e bem-estar social (10 incidências). Ambos os condicionantes indicaram aspectos qualitativos dos relacionamentos durante a fase de consolidação da rede. De acordo com Atouba e Shumate (2010), os resultados encontrados na baixa incidência destes condicionantes podem ser explicados devido ao grau de maturidade dos relacionamentos da rede durante sua consolidação. Na Aprovale, embora a confiança e o bem-estar social representem temas importantes entre os associados, por não estarem diretamente relacionados ao processo produtivo ou com a capacidade de geração de recursos financeiros para o processo de reinvestimento na estrutura da rede, acabaram relegados ao segundo plano.

Sirmon e colaboradores (2010), ao discutirem o processo de coordenação de recursos em redes por meio da teoria da visão baseada em recursos, objetivando a criação de vantagem competitiva, apresentaram conclusões semelhantes aos de Atouba e Shumate (2010) na definição de prioridades estratégicas durante o desenvolvimento de estruturas interorganizacionais. Para Sirmon e colaboradores (2010), a definição dos objetivos estratégicos das redes capazes de promover a criação de vantagem competitiva está relacionada com os seguintes pontos: escopo estratégico do planejamento de recursos da rede, etapas do ciclo de vida das redes e capacidade de inserção das redes nos mercados de atuação. Para esses autores, de acordo com o momento evolutivo e as prioridades estratégicas das redes, os objetivos podem estar concentrados no atendimento de questões de cunho imediato.

Isto significa que, para a rede Aprovale consolidar seu objetivo principal: "criar a primeira Indicação de Procedência (IP) no Brasil para vinhos finos e espumantes", tornou-se necessária ênfase total na orientação de recursos estratégicos dos associados em aspectos produtivos e de diferenciação dos produtos. Portanto, a orientação de condicionantes na consolidação da rede esteve mais voltada ao atendimento de questões estruturais, como a capacidade de gerar caixa para financiamento do próprio processo produtivo. O que explica, dessa forma, o total de incidências dos condicionantes verificados.

No segundo grupo de resultados encontrados, nota-se que a teia de relações formada pelos condicionantes foi polarizada em dois pontos (figura 1): no primeiro ponto, conforme já era esperado, mostram-se concentradas a busca de economias de escala associada com a criação de vantagem competitiva. No segundo ponto, por sua vez, estão alocados o reforço da capacidade produtiva, bem como o reforço da capacidade competitiva das vinícolas envolvidas na consolidação dos objetivos estratégicos da rede. Toda a teia de relações gerada entre os condicionantes está visível na figura 1. 


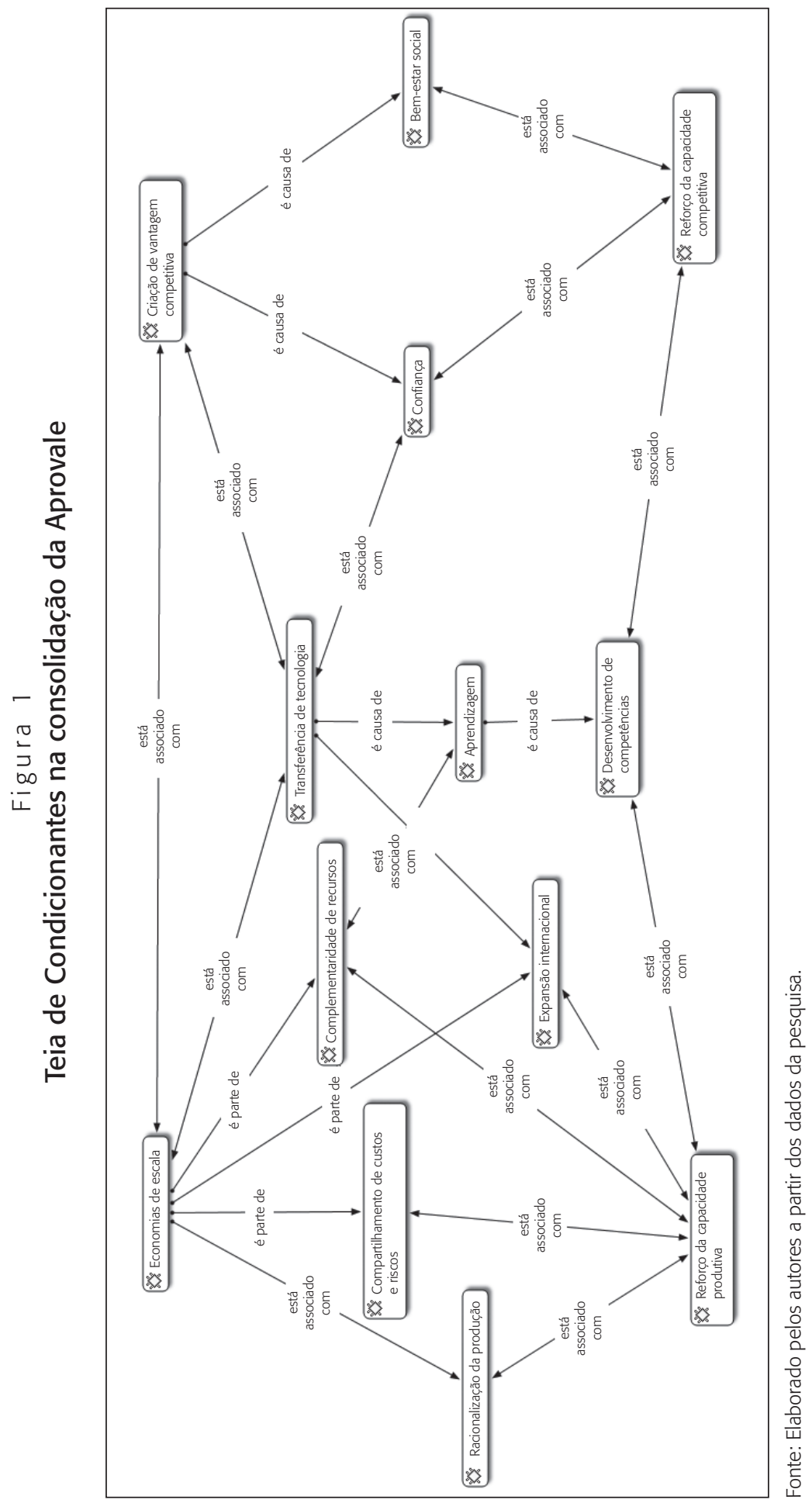


Com base na figura 1, pode-se ressaltar que a busca de economias de escala esteve relacionada com vários condicionantes, entre eles: a racionalização da produção, o compartilhamento de custos e riscos, a complementaridade de recursos, além da expansão internacional. Além disso, os condicionantes considerados nessas relações estiveram também associados diretamente com o reforço da capacidade produtiva dos atores, implicando interdependência entre os relacionamentos de caráter técnico. Na rede, entendem-se como relacionamentos de caráter técnico as atividades compartilhadas entre associados com objetivos de cultivo da uva e processamento industrial de vinhos finos e espumantes. Estes aspectos apontados podem ser verificados em relatos dos respondentes do estudo, a exemplo do que se segue:

Aqui na Aprovale, a partir dos anos 2000, se fala muito em espírito de rede, no qual são buscados integração e complementaridade de recursos entre vinícolas, inclusive, quando o assunto é discutir formas de acesso em novos mercados, por exemplo, o internacional. Na verdade, considero isto importante, mas o que nós fazemos realmente é trabalhar no aumento de nossa capacidade de produção e distribuição, conseguindo com isso diminuir os custos fixos de produção. Como o consumidor brasileiro de vinhos é sensível a preços e a tributação é um entrave do processo produtivo, o jeito encontrado para a manutenção do negócio é a diluição de custos com o aumento da quantidade produzida e vendida (Trecho da entrevista com diretor industrial de uma das vinícolas).

Em outra direção, a criação de vantagem competitiva na teia de relações gerada pode ser caracterizada como a causa de ações que visam o bem-estar social e a construção de processos de confiança nos relacionamentos estabelecidos entre os associados da rede. Uma vez que tanto a confiança como a preocupação com o bem-estar, no contexto de inserção das estruturas interorganizacionais, constituem elementos relacionados com o aumento da capacidade competitiva organizacional (Thorgren e Wincent, 2010). Este aspecto torna esta alternativa interessante à rede, pois demonstra uma alternativa estratégica complementar para a criação de vantagem competitiva, ultrapassando a simples relação com atividades ligadas diretamente aos aspectos produtivos.

Kunzler e Bulgacov (2011), ao verificar metodologias de avaliação de desempenho dos participantes de associações de produtores rurais em Quatro Pontes (PR), chegaram a resultados semelhantes aos encontrados na rede Aprovale, evidenciando a importância de aspectos subjetivos na avaliação de desempenho das redes. Para esses autores, os associados podem usufruir das associações como um meio de melhoria da competitividade e da obtenção de habilidades complementares, acesso a novos mercados, oportunidades para sinergia mútua e aprendizado. Ou seja, a estrutura de relacionamentos que envolvem as associações de produtores rurais é utilizada como instrumento de repasse de recursos oficiais que promovam benefícios econômicos, sociais e informacionais aos associados, viabilizando, mantendo e modernizando a base produtiva.

Na rede estudada, apesar de os condicionantes de cunho qualitativo possuírem importância no segundo plano dos associados, em virtude dos motivos já apresentados neste texto, 
nota-se uma preocupação estratégica da direção da rede em enfatizar tais aspectos no longo prazo. Esta constatação pode ser percebida no relato efetuado pelo gestor da rede.

A preocupação com o bem-estar da comunidade no ambiente de inserção das vinícolas e, consequentemente, da rede, vai além da simples preocupação em atender regulamentos da legislação ambiental. Ao contrário, o principal objetivo neste relacionamento é criar pontos competitivos duradouros no longo prazo, nos quais as atividades produtivas desenvolvidas nas vinícolas passarão a ser consideradas no futuro como um pedaço da paisagem que caracteriza o Vale dos Vinhedos, refletindo a tradição e a cultura da comunidade local (Trecho da entrevista com o representante da Aprovale).

É importante destacar ainda que a transferência de tecnologias na rede Aprovale, durante o processo de consolidação, esteve associada à busca de economias de escala e à criação de vantagens competitivas. Essa associação entre os condicionantes pode ser percebida porque o processo de transferência de tecnologia entre os associados não envolveu somente aspectos relacionados ao processo industrial. Essa prática englobou também elementos que compreenderam toda a dinâmica do negócio da indústria de vinhos finos e espumantes, inclusive as atividades de turismo que foram desenvolvidas em virtude do negócio principal da rede.

Este fato evidencia a percepção de que tanto a busca de economia de escala quanto a criação de vantagens competitivas foram importantes características relacionadas ao processo de transferência de conhecimentos gerados com a atividade de gestão das vinícolas associadas na rede, tenha sido esse conhecimento de cunho operacional ou estratégico. Mediante isso se entende que na Aprovale a transferência de tecnologias pode ser caracterizada como causa importante do processo de aprendizagem dos associados, pois, durante a consolidação da rede, atividades específicas que envolviam os associados configuraram-se, para alguns, como novidades, para outros, como condição essencial para manter competitividade no mercado. Dessa forma, ambas as situações fomentaram um processo de aprendizagem contínuo na rede.

As evidências encontradas nessas situações vivenciadas pelos associados da Aprovale são explicadas a partir de Saeed, Malhotra e Grover (2011), identificando-se duas tendências para explicar o aumento da complexidade tecnológica do novo ambiente no qual os associados da Aprovale foram inseridos durante a consolidação da rede. A primeira das tendências relaciona-se com o aumento da complexidade do processo de produção em termos do número de insumos requeridos, o qual acarretou para os pequenos associados um aumento da utilização de recursos externos, inclusive na área de gestão. Essa tendência refletiu-se no próprio processo produtivo que passou a ser utilizado, cuja estrutura passou a ter maior complexidade.

Uma segunda tendência refere-se ao aumento do conjunto de conhecimentos e competências que necessitaram ser integrados de modo a tornar exequível a produção padrão dos produtos da rede de acordo com as normas da Indicação de Procedência, mantendo a possibilidade da diferenciação desses produtos em face do mercado consumidor. Para Saeed, Malhotra e Grover (2011), a complexidade das tecnologias integradas, ao nível da rede, afeta decisivamente a natureza dos processos de aprendizado gerados em sua estrutura, 
na medida em que impõe determinados requisitos quanto à integração de conhecimentos e competências.

Em particular, na Aprovale, observou-se que determinadas características da base de conhecimentos necessária para gerar diferenciações nos produtos produzidos, durante a fase de consolidação da rede, afetaram diretamente as possibilidades de cooperação entre os associados. A primeira das características envolveu o caráter tácito dos conhecimentos relevantes ao processo produtivo dos vinhos finos e dos espumantes, principalmente aqueles que estavam concentrados em associados expressivos que integravam a rede após sua formação. Vale destacar que, apesar da rede ter sido formada por pequenas empresas em 1995, no momento da consolidação dos objetivos iniciais, grandes grupos já faziam parte dela, inclusive com estrutura multinacional na área de Pesquisa e Desenvolvimento.

Outra característica importante está relacionada com a complexidade da base de conhecimentos existente na Aprovale, especificamente com: a) a diversidade de disciplinas e tecnologias que necessitaram ser integradas de maneira a gerar inovações comuns na rede; b) o conjunto de diferentes competências relacionadas ao processo de produção e a atributos do produto e da demanda. Neste sentido, Posch (2010) destaca que, quanto mais complexa a base de conhecimentos, mais necessário é o desenvolvimento de mecanismos específicos que permitam integrar os vários fragmentos do conhecimento gerados externamente, reforçandose a importância de vínculos cooperativos com outras organizações, bem como de mecanismos de transferência que facilitem essa integração.

Em outra perspectiva, Gottardi e Hens (1996) apresentam que o caráter não complexo dos produtos gerados afeta a intensidade e a orientação dos esforços inovativos realizados nas redes de cooperação de produção agrícola. De fato, esses esforços que aconteceram na estrutura da Aprovale assumiram um caráter não sistemático, envolvendo inovações incrementais baseadas em mecanismos de aprendizado que emergiam como subprodutos das práticas produtivas e dos relacionamentos adotados pelos diferentes associados com organizações internas ou externas à rede.

Desse modo, torna-se evidente que o processo de consolidação da Aprovale esteve necessariamente baseado em um conjunto de interações sistemáticas de aprendizagem entre diversos atores presentes em seu contexto de inserção, entre eles: Instituto Brasileiro do Vinho, Universidade de Caxias do Sul, Serviço Brasileiro de Apoio a Micro e Pequenas Empresas, Empresa Brasileira de Pesquisa Agropecuária. Isso permitiu uma formatação padronizada de conhecimentos perante exigências do processo de aprendizagem necessário para a consolidação das atividades entre seus associados. Essa inclusão de organizações externas à realidade da rede também permitiu a complementaridade de recursos necessários à consecução dos objetivos iniciais da Aprovale, mas não disponíveis internamente.

Por outro lado, nas situações em que os associados demandaram por conhecimentos de maior complexidade, alheios aos objetivos comuns adotados na rede, verificou-se a não padronização dos fluxos informacionais e dos procedimentos organizacionais. No entanto, a estrutura de aprendizagem da rede permaneceu em pleno desenvolvimento, adotando dire- 
cionamentos comuns, apesar das diferenças estratégicas dos associados. Por essa razão, o processo de aprendizagem obtido nesta fase de consolidação da rede esteve também associado com outros condicionantes, entre eles aqueles referentes à expansão internacional, bem como os condicionantes que visavam a complementaridade de recursos em atividades específicas dos associados ou da rede como um todo.

Em síntese, por meio dos resultados obtidos na análise de condicionantes, identificados como responsáveis pela consolidação da rede Aprovale, verificou-se que a obtenção de economias de escala e a criação de vantagem competitiva consistiram nos pontos iniciais que envolveram a dinâmica de consecução desta fase do ciclo de vida da rede analisada. Nessa direção, torna-se salutar destacar que na rede Aprovale a obtenção de economias de escala estava relacionada com atividades técnicas (plantio e produção) e comerciais (distribuição). De outra maneira, relacionados com a criação de vantagem competitiva, estiveram atrelados os condicionantes de caráter mais qualitativo (relacional), como o desenvolvimento de processos de confiança e de bem-estar social.

Conclui-se com isso que, durante a fase de consolidação da rede em questão, os procedimentos técnicos e comerciais estiveram relacionados com o reforço da capacidade produtiva dos associados no estabelecimento de relacionamentos com organizações externas à rede. Dessa maneira, os condicionantes mais qualitativos estiveram vinculados com o reforço da capacidade competitiva, ou seja, por meio de estratégias de desenvolvimento futuro, questões não tão emergentes ganharam foco no desenvolvimento de atividades compartilhadas na rede. Isso permitiu nesse processo a intenção de um reforço competitivo mais sustentável e duradouro do que aqueles construídos somente em termos produtivos e com preocupações de curto prazo.

\section{Considerações finais}

Este trabalho objetivou descrever os condicionantes envolvidos durante o processo de consolidação da rede Aprovale no setor vitivinícola brasileiro. Por isso, com base no conceito de redes percebido como estratégias de cooperação, foram analisadas percepções de gestores dessa rede e de instituições atuantes no setor vitivinícola brasileiro a respeito dos relacionamentos estabelecidos na rede que foram orientados na consecução dos objetivos definidos em sua formação.

Os resultados indicaram, de um modo geral, que a consolidação da rede Aprovale possibilitou o desenvolvimento de seus associados, impactando na forma de atuação das vinícolas junto aos mercados de inserção regional/nacional, seja por inovações nos produtos, nos processos e na forma de gestão ou, ainda, pelas mudanças estabelecidas nas relações entre os associados e entidades representativas do setor vitivinícola.

Na consolidação da rede, a influência de forças do ambiente competitivo sobre os associados, a chegada de multinacionais na Serra Gaúcha e a adoção de novas tecnologias de vini- 
ficação constituíram os marcos definidores do modelo da estrutura organizacional pretendida: a Indicação de Procedência (IP) Vale dos Vinhedos. Portanto, os condicionantes de maior destaque estiveram atrelados em obtenção de economias de escala e a criação de vantagem competitiva, consistindo nos pontos cruciais que envolveram a dinâmica de consecução desta fase na rede analisada. Condicionantes de caráter mais qualitativo (relacionais), como o desenvolvimento de processos de confiança e de bem-estar social, também se fizeram presentes na consolidação da rede, apesar de sua menor expressão na dinâmica percebida. Esses condicionantes qualitativos envolveram questões estratégicas de longo prazo, sendo fomentados por ideais de sustentabilidade e crescimento duradouro da rede.

Os estudos propostos por Ring e Van de Ven (1994) e por Franco (2007) demonstram que o processo de superação de desafios vivenciados pelas redes de cooperação durante sua evolução faz com que os associados modifiquem com sucesso as formas de relacionamentos estabelecidos. Nesta direção, notou-se na consolidação da Aprovale que a incorporação de novos conhecimentos, por meio do compartilhamento e complementaridade de recursos geridos por organizações externas, tornou possível a consecução do objetivo principal da rede, preservando diferenças internas dos associados e seu grau de competitividade. Isso demonstra a principal contribuição teórica deste estudo, ao apontar que o detalhamento de relações, entre os condicionantes de consolidação apresentados, sugere um caminho alternativo para novas pesquisas sobre relacionamentos interorganizacionais que se estruturem de maneira cooperada.

Sugerem-se estudos futuros sobre a análise da ação dos condicionantes verificados no processo de consolidação da rede Aprovale em suas demais fases evolutivas, além da verificação da influência externa que relacionamentos presentes em outros contextos e diferentes níveis de análise possuem no processo de consolidação de redes situadas nos demais setores produtivos brasileiros.

\section{Referências}

APROVALE. Associação dos Produtores de Vinhos Finos do Vale dos Vinhedos. Disponível em: <www.vv.com.br> Acesso em: 13 ago. 2011.

ATOUBA, Yannick; SHUMATE, Michelle. Interorganizational networking patterns among development organizations. Journal of Communication, v. 60, n. 2, p. 293-317, 2010.

BALESTRIN, Alsones; VERSCHOORE, Jorge R.; REYS JR., Edgar. O campo de estudos sobre redes de cooperação interorganizacional no Brasil. RAC, v. 14, n. 4, p. 458-477, 2010.

BLEEKE, Joel; ERNST, David. Collaborating to compete: using strategic alliances and acquisitions in the global marketplace. Nova York: John Wiley \& Sons, Inc., 1992. 
BRASS, Daniel J. et al. Taking stock of networks and organizations: a multilevel perspective. The Academic Management Journal, v. 47, n. 6, p. 795-817, 2004.

CHEN, Shih-Fen S. A general TCE model of international business institutions: market failure and reciprocity. Journal of International Business Studies, v. 41, p. 935-959, 2010.

CRIADO, Alex R.; CRIADO, Josep R. El papel de los acuerdos de cooperación en los processos de internacionalización de la empresa española: un análisis empírico. Papeles de Economia Española, v. 66, p. 248-266, 1996.

EMBRAPA. Empresa Brasileira de Pesquisa Agropecuária: dados técnicos. Disponível em: <www. embrapa.gov.br> Acesso em: 10 out. 2011.

FENSTERSEIFER, Jaime E. The emerging Brazilian wine industry: challenges and prospects for the Serra Gaúcha wine cluster. International Journal of Wine Business Research, v. 19, n. 3, p. 187-206, 2007.

FRANCO, Mário J. B. Tipologia de processos de cooperação empresarial: uma investigação empírica sobre o caso português. RAC, v. 11, n. 3, p. 149-176, 2007.

GLAISTER, Keith W.; BUCKLEY, Peter J. Strategic motives for international alliance formation. Journal of Management Studies, v. 33, n. 3, p. 301-332, 1996.

GOTTARDI, Piero; HENS, Thorsten. The survival assumption and existence of competitive equilibrium when asset markets are incomplete. Journal of Economic Theory, v. 71, n. 2, p. 313-323, 1996.

HAUSMAN, Warren $\mathrm{H}$. et al. A process analysis of global trade management: an inductive approach. Journal of Supply Chain Management, v. 46, n. 2, p. 5-29, 2010.

IBRAVIN. Dados históricos e recentes do setor vitivinícola brasileiro. Disponível em: <www.ibravin. com.br> Acesso em: 10 maio 2011.

KENT, David H. Joint Ventures vs. non-joint ventures: an empirical investigation. Strategic Management Journal, v. 12, n. 5, p. 387-393, 1991.

KUNZLER, Mali T.; BULGACOV, Sergio. As estratégias competitivas e colaborativas e os resultados individuais e coletivos no associativismo rural em Quatro Pontes (PR). Revista de Administração Pública, Rio de Janeiro, v. 45, n. 5, p. 1363-1393, set./out. 2011.

LEE, Seungyoon; MONGE, Peter. The coevolution of multiplex communication networks in organizational communities. Journal of Communication, v. 61, n. 4, p. 758-779, 2011.

MARTES, Ana C. B. et al. Fórum - redes sociais e interorganizacionais. Revista de Administração de Empresas, v. 46, n. 3, p. 10-15, 2006.

MERCHANT, Hemant; SCHENDEL, Dan. How do international joint ventures create shareholders value? Strategic Management Journal, v. 21, n. 7, p. 723-737, 2000.

MOWERY, David C.; OXLEY, Joanne E.; SILVERMAN, Brian S. Strategic alliances and interfirm knowledge transfer. Strategic Management Journal, v. 17, Special Number, p. 77-91, Winter 1996. 
MÜLLER-SEITZ, Gordon. Leadership in interorganizational networks: a literature review and suggestions for future research. International Journal of Management Reviews, v. 14, n. 4, p. 428-433, 2011.

MURRAY, Janet Y. Patterns in domestic vs. international strategic alliances: an investigation of US multinational firms. Multinational Business Review, v. 3, n. 2, p. 7-16, 1995.

OHMAE, Kenichi. Pour quoi les alliances échouent-elles? Harvard L'Expansion, v. 2, n. 56, p. 25-44, 1990.

PARK, Seung H.; UNGSON, Gerardo R. The effect of national culture, organizational complementarity, and economic motivation on joint venture dissolution. Academy of Management Journal, v. 40, n. 2, p. 279-307, 1997.

PERROW, Charles. Small-firms networks. In: NOHRIA, Nitin; ECCLES, Robert G. (Ed.). Networks and organizations: structure, form, and action. Cambridge: Harvard University Press, 1992.

POLETTO, Carlos A.; DUARTE, Maria A.; MATA, Wilson. Gestão compartilhada de P\&D: o caso da Petrobras e a UFRN. Revista de Administração Pública, Rio de Janeiro, v. 45, n. 4, p. 1095-1117, set./out. 2011.

POSCH, Alfred. Industrial recycling networks as starting points for broader sustainability-oriented cooperation? Journal of Industrial Ecology, v. 14, n. 2, p. 242-257, 2010.

POWELL, Walter W. Learning from collaboration: knowledge and networks in the biotechnology and pharmaceutical industries. California Management Review, v. 40, n. 3, p. 228-240, 1998.

RICKS, James E. Benefits of domestic vertical and horizontal strategic alliances: to compete with international cartels and the Japanese keiretsu. Journal of Business \& Industrial Marketing, v. 8, n. 4, p. 52-57, 1993.

RING, Peter S.; VAN DE VEN, Andrew H. Developmental processes of cooperative interorganizational relationships. Academy of Management Journal, v. 19, n. 1, p. 90-118, 1994.

SACHWALD, Frédérique. Cooperative agreements and theory of the firm: focusing on barriers to change. Journal of Economic Behavior \& Organization, v. 35, n. 2, p. 203-225, 1998.

SAEED, Khawaja A.; MALHOTRA, Manoj K.; GROVER, Varun. Interorganizational system characteristics and supply chain integration: an empirical assessment. Decision Sciences, v. 42, n. 1, p. 7-42, 2011.

SIRMON, David G. et al. Capability strengths and weaknesses in dynamic markets: investigating the bases of temporary competitive advantage. Strategic Management Journal, v. 31, n. 13, p. 13861409, 2010.

STEENSMA, H. K. et al. The influence of national culture on the formation of technology alliances by entrepreneurial firms. Academy of Management Journal, v. 43, n. 5, p. 951-973, 2000. 
THOMPSON, Grahame F. Between hierarchies and markets: the logic and limits of network form of organization. Nova York: Oxford University Press, 2003.

THORGREN, Sara; WINCENT, Joakim. Interorganizational trust: origins, dysfunctions and regulation of rigidities. British Journal of Management, v. 22, n. 1, p. 21-41, 2010.

TURRINI, Alex et al. Networking literature about determinants of network effectiveness. Public Administration, v. 88, n. 2, p. 528-550, 2009.

Claudio Zancan é doutor em administração e professor adjunto na Faculdade de Economia, Administração e Contabilidade da Universidade Federal de Alagoas (Feac/Ufal). E-mail: claudiozancan@gmail.com.

Paulo da Cruz Freire dos Santos é doutor em engenharia de produção e professor associado na Faculdade de Economia, Administração e Contabilidade da Universidade Federal de Alagoas (Feac/Ufal). E-mail: paulodacruzfreire@gmail.com.

Antônio Carlos Silva Costa é doutor em psicologia experimental e professor associado na Faculdade de Economia, Administração e Contabilidade da Universidade Federal de Alagoas (Feac/Ufal). E-mail: acscosta@uol.com.br.

Nicholas Joseph Tavares da Cruz é doutorando em engenharia de produção e professor assistente na Faculdade de Economia, Administração e Contabilidade da Universidade Federal de Alagoas (Feac/Ufal). E-mail: admnicholas@gmail.com. 
